\title{
Comparative study of the effect of obesity on lipid profiles levels, insulin resistance and oxidative stress in adolescents
}

\author{
N. Sundaraveena ${ }^{1}$, T. Santhi ${ }^{2}$, T. Uma ${ }^{1 *}$ \\ ${ }^{1}$ Department of Biochemistry, Rajiv Gandhi Institute of Medical Sciences, Srikakulam, Andhra Pradesh, India, \\ ${ }^{2}$ Department of Biochemistry, NRI Medical College, Sangeevalasa, Andhra Pradesh, India.
}

*Address for Correspondence: T. Uma, Email: t $\underline{\text { daffadils@ yahoo.co.in }}$

\begin{abstract}
Introduction: Obesity is associated with social and medical risks that especially make it a problem. The study is to determine whether the lipid abnormalities observed in obese adolescents are associated with insulin resistance \& oxidative stress. Methods: We evaluated the relationship between lipid levels and insulin resistance and oxidative stress in 30 female obese adolescents who were obese (body mass index $>25 \mathrm{Kg} / \mathrm{m}$ ). Insulin resistance was assessed by fasting insulin and glucose level, Lipid profile was studied including, total cholesterol, HDL, LDL, and oxidative stress by estimating vitamin-c and Malandialdehyde. Results: All the parameters except serum HDL level showed significant increase in obese adolescents persons while HDL level was significantly decreased. Conclusion: The study indicates elevation of glucose, lipid profile levels and oxidative stress in obese adolescents. This is due to hyperinsulinaemia leads to insulin resistance. Insulin resistance may be responsible, for changes in lipid and lipoprotein concentration. childhood weight requires special early attention because insulin resistance, inflammation and dyslipidemia increase the risk of cardiovascular disease
\end{abstract}

Keywords: Oxidative Stress, Insulin Resistance, Dyslipidemia, Malandialdehyde

\section{Introduction}

Obesity is rapidly becoming one of the most important medical and public health problems of our time. Obesity is associated with a high rate of morbidity and early mortality if left untreated [1]. Therefore, obesity is currently identified as a major health problem. Although obesity in childhood and adolescence is more common among countries with high economic standards, its prevalence is growing rapidly in developing countries [2] .Obesity is a chronic disease with multifactorial etiology. In childhood and adolescence, it often leads to important metabolic alterations, which, depending on the duration and severity, may determine the development of chronic diseases in adulthood [3,4]. Obese individuals often present with metabolic disorders, such as high blood pressure, elevated fasting glucose levels and lipid abnormalities, which promote vascular injury and endothelial dysfunction [5]. Lipid abnormalities have also been reported in obese adults, who have elevated

Manuscript received: $17^{\text {th }}$ Aug 2015

Reviewed: $29^{\text {th }}$ Aug 2015

Author Corrected: $10^{\text {th }}$ Sept 2015

Accepted for Publication: $17^{\text {th }}$ Sept 2015 triglycerides and LDL cholesterol and low levels of HDL cholesterol. Similar lipid profiles have been reported in obese and nonobese adults with type 2 diabetes, in obese normoglycemic adults, and in nonobese adults with impaired glucose tolerance [6,7]. As the central dogma regarding the pathophysiology of obesity unfolds, it is evident that the expansion of visceral adipose tissue caused by overconsumption of nutrients plays a central role. As visceral fat stores expand, adipocytes generate increasing levels of reactive oxygen species (ROS) that incite increased expression and secretion of inflammatory adipokines .Oxidative stress leads to insulin resistance within adipose tissue as well as in peripheral tissues. Insulin resistance is one of the hallmarks of obesity and accounts for many of its comorbidities, including hypertension [8]. To determine whether the lipid abnormalities observed in obese adolescents are associated with insulin resistance and oxidative stress.

\section{Material and Methods}


This study was conducted in 2014. It included 10-18 year-old children and female adolescents (30) who had been attended to out patient to the Pediatric department of RIMS hospital srikakulam. Children with diabetes, thyroid diseases, any disease related to hepatic problems, or any pharmacological treatment that could lead to changes in blood lipids were excluded from the study. The study protocol complied with Helsinki Declaration guidelines and was approved by the Clinical Research Ethics Committee of the RIMS. After explaining the objectives of the study to the participants, written consents were obtained. Parents were also required to sign a written consent form for participation of their children in the study.

Measurements were taken with the children anthropometric and blood tests. The examinations were conducted by a professional team including a physician, a trained nurse, and a pediatrician. Anthropometric evaluations included measuring height and weight to calculate body mass index (BMI). Height was measured to the nearest millimeter using a portable stadiometer, and weight was recorded to the nearest $0.1 \mathrm{~kg}$ using a standardized electronic digital scale while the subjects were wearing light clothing and barefoot. From these measurements, BMI (weight in kilograms divided by height in meters squared) was computed.

Fasting (12 hours) venous blood samples were obtained by venipuncture. Plasma total cholesterol, HDL cholesterol, triglyceride, glucose, and insulin levels were measured were measured using a enzymaticphotometric analyzer using commercial kits. LDL cholesterol was calculated using the Friedewald formula [9] The homeostatic model assessment index (HOMA index $=$ fasting glucose $[\mathrm{mmol} / \mathrm{L}] \times$ fasting insulin $[\mu \mathrm{U} / \mathrm{mL}]$ / 22.5) was used as a measure of insulin sensitivity [10].

we considered children to be obese or overweight if their BMI exceeded the age- and sex-specific cut-off points [11]. .A Student's t test analysis was used to compare the mean values between controls \& obese adolescents . Statistical analyses were performed using the SPSS software package, version 14.0.All data was recorded in special forms and reported as mean \pm standard deviation (SD). For all analyses, statistical significance was considered as $\mathrm{p}<0.05$.

\section{Results}

The biochemical findings of this study are expressed in the form of the following results. The results were expressed as Mean \pm SD. The normal values are compared to the patient value. For all parameter of the study, the mean and standard deviation were calculated for patient and control subjects. Glucose and lipid profile levels were estimated in both Controls \& Patients. 30 normal are taken as control subjects who are non obese 30 obese are taken as case subjects who are female obese aldolescents for the study.

Glucose values in obese FBS (113.6 \pm 46$)$ elevated compared to control subjects (78 \pm 9$)$, Glucose values in obese PPBS (132 \pm 9$)$ elevated compared to control subjects $(116 \pm 13)$, cholesterol values in obese $(249 \pm 19)$ elevated compared to control subjects $(187 \pm 34)$, TgL values in obese $(199 \pm 46)$ elevated compared to control subjects (135 \pm 29$)$, LDL values in obese $(132 \pm 18)$ elevated compared to control subjects $(107 \pm 29)$, HDL values in obese (46 \pm 12$)$ decreased compared to control subjects $(54 \pm 11)$ Insulin values in obese FBS (27.0 \pm 5.2$)$ elevated compared to control subjects (18.4 \pm 3.4$)$, HOMA-IR values in obese FBS (7.5 \pm 1.7$)$ elevated compared to control subjects $(3.5 \pm 0.7)$, Oxidative Stress is higher than controls. i.e MDA as oxidant in obese (6.0 \pm 2.1$)$ elevated compared to control subjects $(4.3 \pm 1.7)$, vitamin-c as antioxidant in obese $(0.7 \pm 0.5)$ elevated compared to control subjects $(1.2 \pm 0.5)$.

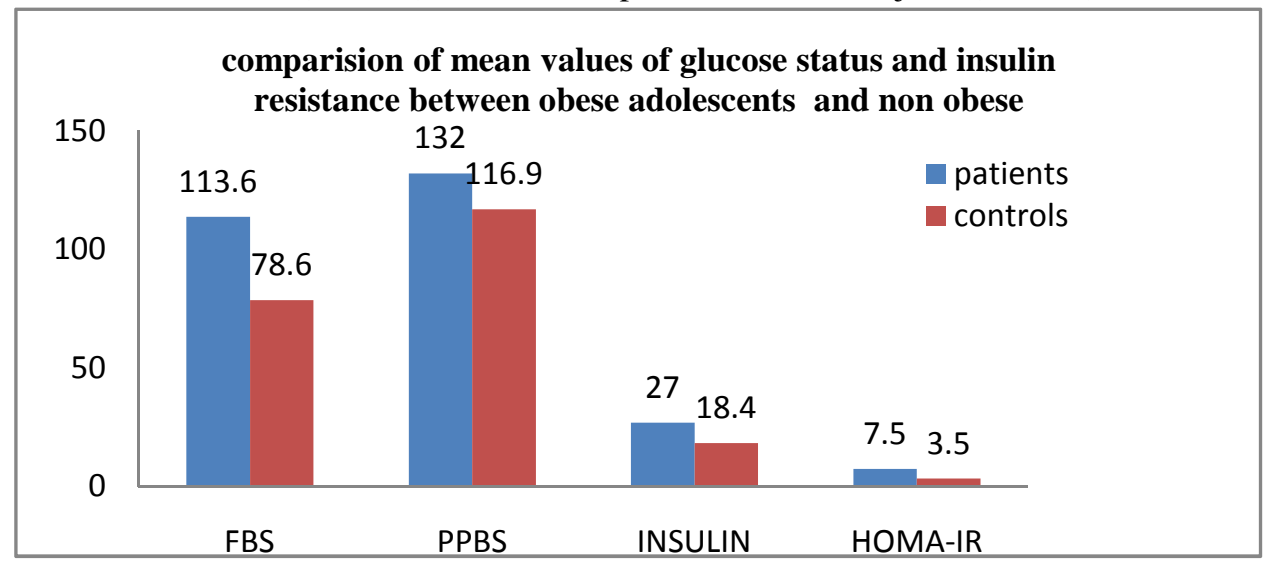


Table 1: Showing the comparative mean values of obese adolescents and control

\begin{tabular}{|l|l|l|l|l|l|}
\hline & & \multicolumn{2}{l|}{ Obese adolescents } & \multicolumn{2}{l|}{ Controls } \\
\hline S. No & Parameters & Mean & SD & Mean & SD \\
\hline 1 & FBS & 113.6 & 17.41 & 78.6 & 9.5 \\
\hline 2 & PPBS & 132 & 9.3 & 116.9 & 13.6 \\
\hline 3 & INSULIN & 27.0 & 5.2 & 18.4 & 3.4 \\
\hline 4 & HOMA-IR & 7.5 & 2.7 & 3.5 & 0.7 \\
\hline 5 & Cholesterol & 249.8 & 21.1 & 187 & 34.2 \\
\hline 6 & Triglycerides & 199.9 & 46.8 & 135 & 29.4 \\
\hline 7 & HDL & 46.5 & 12 & 54 & 11.2 \\
\hline 8 & LDL & 132.1 & 18.1 & 107.8 & 29.9 \\
\hline 9 & MDA & 6.0 & 2.1 & 4.3 & 1.7 \\
\hline 10 & vit-c & 1.2 & 0.5 & 0.7 & 0.5 \\
\hline 11 & age & 11.3 & 0.6 & 10.5 & 0.5 \\
\hline 12 & BMI & 94.2 & 0.5 & 75.2 & 2.4 \\
\hline
\end{tabular}
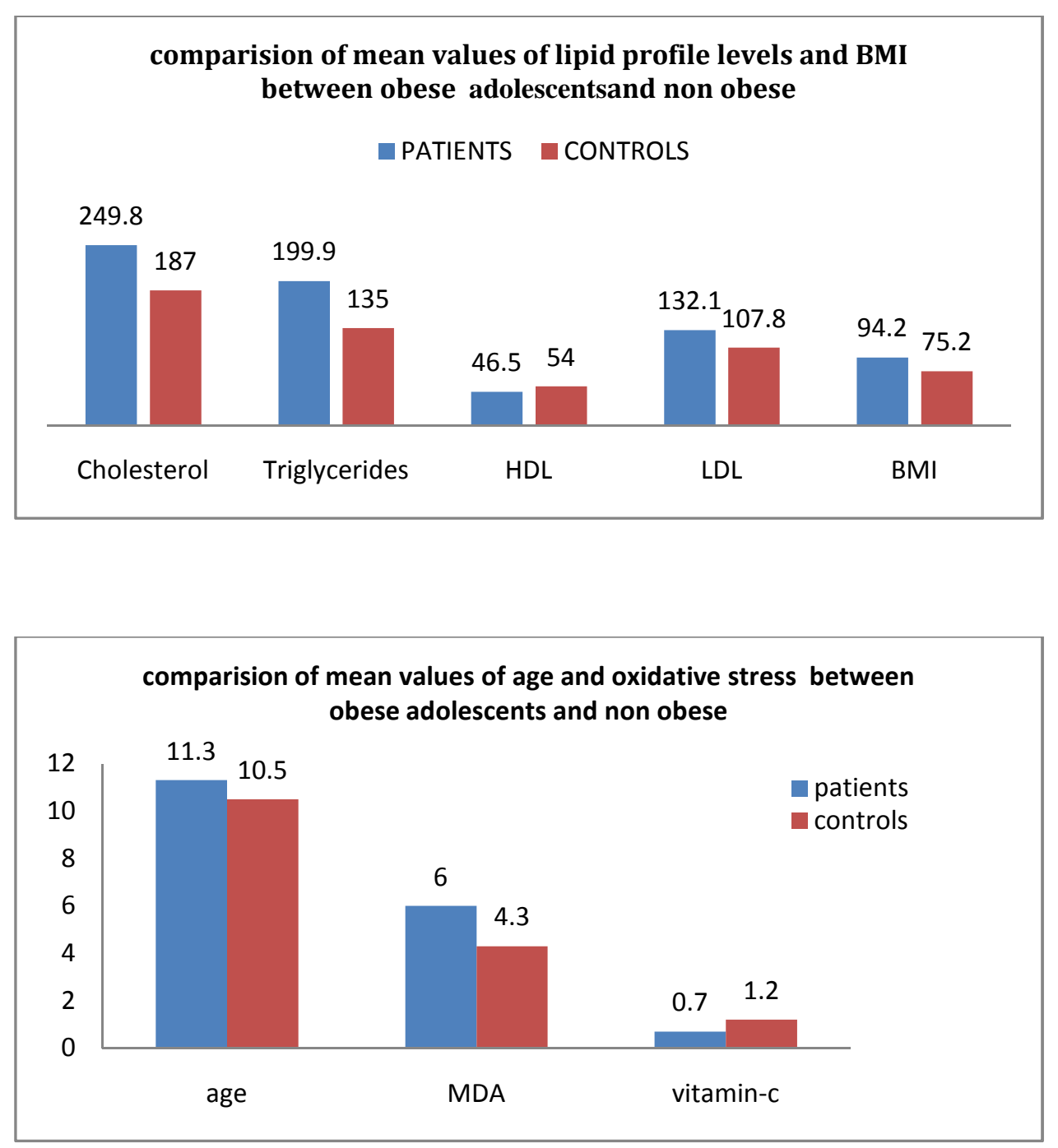


\section{Discussion}

Obesity, Dyslipidemia and Diabetes were considered as independent risk factors for coronary vascular disease and is associated with high amount of morbidity and mortality. Even though they were independent risk factors, the three entities were closely related i.e. obesity leads to insulin resistance which in turn causes type 2 diabetes and both together leads to dyslipidemia [12].

Serrano et al. observed that overweight adolescents were 4.5-fold more likely to have alterations in HOMAIR index and that these values were higher in adolescents with higher percentage of body fat:[13] Costa et al. investigated the clustering of cardiovascular risk factors in 118 children and adolescents according to BMI quartiles and found that being overweight was associated with increased blood pressure, triglycerides, HOMA-IR index, and low HDL-C, comprising a proatherosclerotic profile in this population.[14]. The aim of the study was to determine abnormal glucose, lipid profiles. obese adolescents had higher fasting glucose, and higher TC, LDL, TGL and lower HDL values .When various parameters of blood lipid profile were compared it was observed that there was significant difference between obese and non-obese subjects in relation to total cholesterol, triglycerides and high density lipoprotein. It is important to point out that obesity or distribution of fat exerts effect on blood lipids, specifically as increased triglycerides and cholesterol, reduced HDL cholesterol .On the average, the more fat, the more likely an individual will be dyslipidemic. Increased triglycerides level in obese impaired lipolysis which reduced the HDL-C concentrations.Estrogens affect fat cells directly or through estrogen receptors, and promote fat tissue deposition. Evidence suggests that estrogen increases the differences in adipose tissue depots via subcutaneous lipid accumulation in woman and visceral fat deposition in men [15].

Obesity is a very common cause of insulin resistance .In obese individuals, adipose tissue releases increased amounts of non-esterified fatty acids, glycerol, hormones, pro-inflammatory cytokines and other factors that are involved in the development of insulin resistance. When insulin resistance is accompanied by dysfunction of pancreatic islet $\beta$-cells that release insulin leads in failure to control blood glucose levels.
In obese individuals, increased glucose a level is due to hyperinsulinaemia leads to insulin resistance may be responsible, for changes in lipid and lipoprotein concentration. Lipid mobilization from the fat depots and release of FFA is mainly regulated by catecholamines and insulin (16). Fig:1 Several mechanisms whereby insulin resistance could cause an alteration in lipid metabolism have been described. Hyperinsulinemia is known to enhance hepatic verylow-density lipoprotein synthesis and thus may directly contribute to the increased plasma triglyceride and LDL cholesterol levels [17]. Resistance to the action of insulin on lipoprotein lipase in peripheral tissues may also contribute to elevated triglyceride and LDL cholesterol levels $[18,19]$.

Obesity is also associated with chronic inflammatory response characterized by altered cytokine production and activation of inflammatory signaling pathways. Recent reports have linked this inflammatory response to the development of insulin resistance in 2 different ways. First, activation of inflammatory signaling intermediates may be directly involved in serine phosphorylation of IRS-1 within insulin-sensitive cell types such as hepatocytes and myocytes and thereby in inducing insulin resistance. Second, inflammatory cell infiltration within adipose tissue may be involved in altering adipocyte lipid metabolism (for example, tumor necrosis factor- $\alpha[$ TNF- $\alpha]$ is reported to promote lipolysis) as well as altering cytokine production by adipose tissue, which may in turn have downstream effects in other metabolically important tissues:[20]. As visceral fat stores expand, adipocytes generate increasing levels of reactive oxygen species (ROS) that incite increased expression and secretion of inflammatory adipokines (1-3). ROS occur under physiological conditions and in many diseases and cause direct or indirect damage in different organs; thus, it is known that oxidative stress (OS) is involved in pathological processes such as obesity, diabetes, cardiovascular disease, and atherogenic processes. It has been reported that obesity may induce systemic OS and, in turn, OS is associated with an irregular production of adipokines, which contributes to the development of the metabolic syndrome [21]. In fact, the present study confirms the literature data by showing that IR is present in obese children and adolescents, and that this condition is associated with clinical and metabolic alterations. 
Fig:1

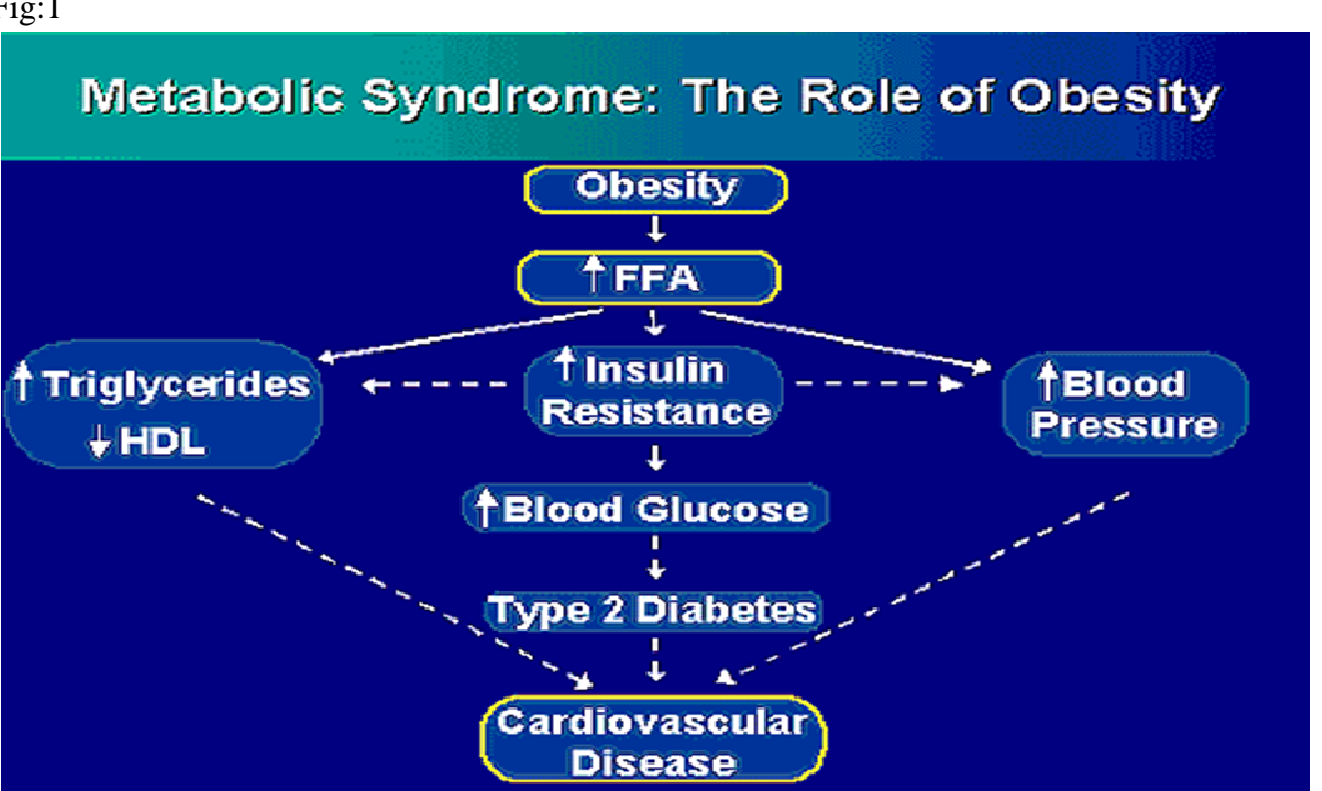

\section{Conclusion}

The past decade has provided a framework for understanding of the functions of adipose tissue in metabolic disease. The role of adipose tissue as a dominant regulator of whole-body lipid and glucose homeostasis is now well established, based on extensive experimental evidence, which shows that dysfunctions in adipose tissue metabolism have a direct impact on lipid and glucose homeostasis. Indeed, the combination of hyperphagia and adipose dysfunction seems to underlie important metabolic pathologies, such as insulin resistance, type 2 diabetes and cardiovascular diseases. Adipose dysfunctions in obesity include secretions of abnormal levels of cytokines linked to insulin resistance, impairments in triglyceride storage and increases in lipolysis [22]. The study indicates elevation of glucose and lipid profile levels in obese adolescents this is due to hyperinsulinaemia leads to insulin resistance. Insulin resistance may be responsible, for changes in lipid and lipoprotein concentration. Lipid mobilization from the fat depots and release of FFA .childhood weight requires special early attention because insulin resistance, inflammation and dyslipidemia increase the risk of cardiovascular disease in adults. We also found that lipid, obesityinflammation, and insulin sensitivity domains predominantly exist in the data of obese children. These domains might be applied to predict the outcomes of cardiovascular diseases in the future. The association of obesity with the insulin resistance syndrome and cardiovascular risk is not only related to the degree of obesity but also seems to be critically dependent on body fat distribution.

\section{Funding: Nil \\ Conflict of interest: None. \\ Permission of IRB: Yes}

\section{References}

1. Plourde G. Impact of obesity on glucose and lipid profiles in adolescents at different age groups in relation to adulthood. BMC Fam Pract. 2002 Oct 14;3:18.

2. Roya Kelishadi, Mahin Hashemipour, Ahmad Sheikh-Heidar, and Shohreh Ghatreh-Samani. Changes in serum lipid profile of obese or overweight children and adolescents following a lifestyle modification courseARYA Atheroscler. 2012 Fall; 8(3): 143-148.

3. Deshmukh-Taskar P, Nicklas TA, Morales M, Yang SJ, Zakeri I, Berenson GS. Tracking of overweight status from childhood to young adulthood: the Bogalusa Heart Study. Eur J Clin Nutr. 2006 Jan;60(1):48-57.

4. P.L. Mendes, M.S. Maciel, L.F. Pinto, D.O. Souza, L.S. Conti, B.V. Mello, et al. The child-adolescent obesity and the impact from childhood to adult life: a systematic review of scientific literature in the period 1997-2007 Pediatria (São Paulo)., 31 (2009), pp. 260273.

5. Vykoukal D, Davies MG. Biology of metabolic syndrome in a vascular patient. Vascular. 2012 
Jun;20(3):156-65. doi: 10.1258/vasc.2011.201201. Epub 2012 Jun 14.

6. Evans DJ, Hoffmann RG, Kalkhoff RK, Kissebah AH. Relationship of body fat topography to insulin sensitivity and metabolic

profiles in premenopausal women. Metabolism. 1984 Jan;33(1):68-75.

7. Krotkiewski M, Bjorntorp $\mathrm{P}$, Sjostrom L, et al. Impact of obesity on metabolism in men and women: importance of regional adipose tissue distribution. J Clin Invest. 1983 Sep;72(3):1150-62.

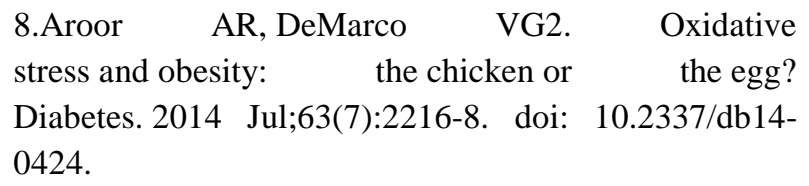

9.Friedewald WT, Levy RI, Fredrickson DS. Estimation of the concentration of low-density lipoprotein cholesterol in plasma, without use of the preparative ultracentrifuge. Clin Chem. 1972 Jun;18(6):499-502.

10.Matthews DR, Hosker JP, Rudenski AS, Naylor BA, Treacher DF, Turner RC. Homeostasis model assessment: insulin resistance and beta-cell function from fasting plasma glucose and insulin concentrations in man. Diabetologia. 1985 Jul;28(7):412-9.

11. Physical status: the use and interpretation of anthropometry. Report of a WHO Expert Committee. World Health Organ Tech Rep Ser. 1995;854:1-452.

12. Nitesh Mishra, Manoj Kumar Sharma. Central obesity and lipid profile in north indian males. International Journal of Applied Biology and Pharmaceutical Technology vol-3,issue-3,july-sep20121.

13.Serrano HM, Carvalho GQ, Pereira PF, Peluzio Mdo C, Franceschini Sdo C, Priore SE. [Body composition, biochemical and clinical changes of adolescents with excessive adiposity]. Arq Bras Cardiol. 2010 Oct;95(4):464-72. Epub 2010 Aug 13.
14.Costa GB, Horta N, Resende ZF, Souza G, Barreto LM, Correia LH, Nascimento TA, Rios CB, BarretoFilho JA, Lopes HF. Body mass index has a good correlation with proatherosclerotic profile in children and adolescents. Arq Bras Cardiol. 2009 Sep;93(3):2617.

15. Palmer BF, Clegg DJ. The sexual dimorphism of obesity. Mol Cell Endocrinol. 2015;402C:113-119. doi: 10.1016/j.mce.2014.11.029.

16. Plourde G. Impact of obesity on glucose and lipid profiles in adolescents at different age groups in relation to adulthood BMC Family Practice. BMC Fam Pract. 2002 Oct 14;3:18.

17. Stalder M, Pometta D, Suenram A. Relationship between plasma insulin levels and high density lipoprotein cholesterol levels in healthy men. Diabetologia. 1981 Dec;21(6):544-8.

18. Pykälistö OJ, Smith PH, Brunzell JD. Determinants of human adipose tissue lipoprotein lipase. Effect of diabetes and obesity on basal- and diet-induced activity. J Clin Invest. 1975 Nov;56(5):1108-17.

19. Sadur CN, Yost TJ, Eckel RH. Insulin responsiveness of adipose tissue lipoprotein lipase is delayed but preserved in obesity. J Clin Endocrinol Metab. 1984 Dec;59(6):1176-82.

20. Savage DB, Petersen KF, Shulman GI. Mechanisms of insulin resistance in humans and possible links with inflammation. Hypertension. 2005 May;45(5):828-33. Epub 2005 Apr 11.

21. Esposito K, Ciotola M, Schisano B, Misso L, Giannetti G, Ceriello A, Giugliano D. Oxidative stress in the metabolic syndrome. $\mathrm{J}$ Endocrinol Invest. 2006 Oct;29(9):791-5.

22. Guilherme A, Virbasius JV, Puri V, Czech MP. Adipocyte dysfunctions linking obesity to insulin resistance and type 2 diabetes. Nat Rev Mol Cell Biol. 2008 May;9(5):367-77. doi: 10.1038/nrm2391.

\section{How to cite this article?}

N. Sundaraveena, T. Santhi, T. Uma. Correlation of maternal age with placenta previa. Int J Med Res Rev 2015;3(9):919924. doi: 10.17511/ijmrr.2015.i9.172. 